\title{
Effects of partial tonsillectomy on the immune functions of children with obstructive sleep apnea-hypopnea syndrome at early stage
}

\author{
Z.Y. Dai ${ }^{1 *}$, D.Y. Huang ${ }^{2 *}$ and C.Y. Zhou ${ }^{1}$ \\ ${ }^{1}$ Department of Otolaryngology, \\ The First Affiliated Hospital of PLA General Hospital, Beijing, China \\ ${ }^{2}$ Department of Otolaryngology-Head and Neck Surgery, \\ Chinese PLA General Hospital, Beijing, China \\ *These authors contributed equally to this study. \\ Corresponding author: C.Y. Zhou \\ E-mail: chengyongzhoucn@163.com
}

Genet. Mol. Res. 13 (2): 3895-3902 (2014)

Received June 6, 2013

Accepted November 29, 2013

Published January 24, 2014

DOI http://dx.doi.org/10.4238/2014.January.24.15

\begin{abstract}
The purpose of this study was to investigate the changes in the humoral and cellular immunity of children with obstructive sleep apnea-hypopnea syndrome and hypertrophy of tonsils before and after plasma-mediated temperature-controlled radiofrequency ablation treatment. Fifty-seven children suffering from obstructive sleep apnea-hypopnea syndrome and with hypertrophy of tonsils were enrolled in this study. Thirty-seven children were grouped in the partial tonsillectomy group and 20, in the tonsillectomy group. The levels of $\mathrm{CD}^{+}, \mathrm{CD}^{+}, \mathrm{CD}^{+}$, and $\mathrm{CD}^{+} / \mathrm{CD}^{+}$were measured for cellular immunity, and the levels of IgG, IgA, and IgM were measured for humoral immunity. Blood samples were collected before and 1 and 3 months after the operation. The IgG, IgA, and IgM levels in the tonsillectomy group were significantly decreased 1 month after the operation, and recovered to the normal levels within 3 months of the operation $(\mathrm{P}<0.05)$. However, the levels of $\operatorname{IgG}, \operatorname{IgA}$, and $\operatorname{IgM}$ in the
\end{abstract}


partial tonsillectomy group decreased slightly, without a significant difference $(\mathrm{P}>0.05)$. The cellular immunity of the 2 groups was not statistically different pre- and post-operation $(\mathrm{P}>0.05)$. The results from the present study indicate that partial tonsillectomy by plasmamediated temperature-controlled radiofrequency ablation did not impact on the humoral and cellular immunity of children.

Key words: Obstructive sleep apnea-hypopnea syndrome; Immune; Plasma-mediated temperature-controlled radiofrequency ablation; Children

\section{INTRODUCTION}

Obstructive sleep apnea-hypopnea syndrome (OSAHS) in children refers to a series of pathophysiological changes due to the frequent occurrence of partial or complete obstruction of the upper airway during sleep, which disrupts normal ventilation as well as the sleep architecture of children. This condition may lead to behavioral and nervous system-related damages (Lal et al., 2012; Landau et al., 2012). The "gold standard" for the diagnosis of OSAHS is overnight polysomnography (AASM, 2007) in the sleep laboratory. Childhood apnea hypopnea index (AHI) is different from adult AHI in that it has no definite correlation with the other clinical variables considered (irritability, attention deficit disorder, enuresis, hyperactivity, snoring, daytime sleepiness, night sweats, and nocturnal breathing pauses), but it is closely correlated with tonsillar size. Therefore, hypertrophic tonsil management constitutes an important part of the surgical treatment of childhood OSAHS. Adenotonsillectomy is an effective treatment in children with OSAHS and adenotonsillar hypertrophy, which improves the clinical symptoms; prevents long-term complications such as poor performance at school, delayed growth, and Cor pulmonale; and improves the objective respiratory data before and after surgery. The success rate of surgical treatment of OSAHS in children is about $80 \%$ (Brietzke and Gallagher, 2006; Lim and McKean, 2009).

Tonsils are important constituents of the upper respiratory tract defense system that protects from pathogen invasion, as well as a part of Waldeyer's ring. They are immunologically reactive lymphoid organs, which are responsible for the manifestation of specific antibodies and B- and T-cell activity in response to a variety of antigens. Thus, they are involved in humoral and cellular immunity (Zielnik-Jurkiewicz and Jurkiewicz, 2002). The crypt system is a specialized structure of the tonsils (Abbey and Kawabata, 1988). The crypt is subject to a complex pipeline network in which the branches are contained and branch anastomosis extensively exists, in particular, at the tonsillar centers. With respect to their function, tonsillar crypts are more than secretory organs; they possibly retain passing antigens for handling by the immune system. Tonsils have an important immune function. Adenotonsillectomy causes the loss of the immunologic barrier within a short time after surgery, thereby leading to changes in immune functions in children (Goldbart et al., 2006). The levels of IgG, IgA, and IgM in patients undergoing tonsillectomy noticeably decrease 1 month after surgery compared to those in age-matched healthy controls (Kaygusuz et al., 2009).

Low-temperature plasma radiofrequency ablation can be technically used to remove a part of the tonsils and to conduct punching ablation on the remaining part of the tonsil, thus achieving the goal of reducing tonsil volume and reserving the immune function. Although this technique only causes minor tissue damage, such a surgical pattern will destroy the ana- 
tomical structure of the tonsils. Its effects on the immune function of the tonsils have not been confirmed yet. From January 2009 to January 2010, our department applied low-temperature plasma radiofrequency ablation to treat 57 pediatric patients with OSAHS accompanied by tonsillar hypertrophy, of which 37 patients were treated with partial adenoidectomy along with tonsil partial nephrectomy, 20 were treated with low-temperature plasma adenoidectomy of tonsils. We then compared the changes in humoral and cellular immune functions before and after the operation to accumulate the results.

\section{MATERIAL AND METHODS}

\section{Inclusion criteria}

1) Diagnostic criteria for pediatric OSAHS (Marcus et al., 2012): polysomnography was used for nocturnal sleep monitoring, and the breathing disorder time was recorded once if the oronasal airflow stops or if it reduces by $50 \%$ or above from the basic level to more than $6 \mathrm{~s}$; the diagnostic criterion of pediatric OSAHS was defined as an AHI $>5$ times $/ \mathrm{h}$ and/ or an apnea index $>1$ time/h, with the lowest oxygen saturation $\left(\mathrm{SaO}_{2}\right)<92 \%$. 2) In the nasopharyngeal lateral radiographs, an $\mathrm{A} / \mathrm{N}$ ratio $\geq 0.71$ is the criterion for pathological adenoid hypertrophy (Fujioka et al., 1979). 3) Physical examination showed that the bilateral tonsils were beyond the velopharyngeal bow, which was defined as grade III. 4) All children underwent paranasal sinus computed tomography to exclude chronic sinusitis. They confirmed to lack a history of allergic rhinitis, recurrent acute tonsillitis, chronic respiratory diseases, nephritis, and systemic allergic diseases, and upper respiratory tract infection 2 weeks prior to the operation.

\section{Subjects}

According to the case inclusion criteria, children with OSAHS were randomly divided into 2 groups: a group of partial resection of tonsils comprising 37 cases ( 24 males and $13 \mathrm{fe}$ males, aged 2-12 years, with a median age of 5.0 years), and a group of complete resection of tonsils comprising 20 patients (14 males and 6 females, aged 2-10 years, with a median age of 4.6 years). This study was conducted in accordance with the Declaration of Helsinki and was approved by the Ethics Committee of PLA General Hospital. Written informed consent was obtained from all participants.

\section{Surgical methods}

All patients received general anesthesia with tracheal intubation, and EN Tec Coblator plasma surgery systemic therapy instrument (ArthroCareare, US) was used to conduct adenoidectomy under a nasal endoscope. For the partial resection group, ReFlex70 knife was used to remove the internal one-third to one-half portion of the bilateral tonsils and the remaining part of grade I-II would be handled by punching ablation on the upper, middle, and lower poles. During the procedure, the output power was set to 5 speed, which would be sustained for 10-15 s. For the complete resection group, a ReFlex70 knife was used to completely remove the bilateral tonsils along the tonsil capsules. 
After the operation, the patients were shifted to the post-anesthesia care unit, and then transferred back to their wards after regaining consciousness. The patients were discharged after 3 days of anti-infection treatment. The patients were suggested to gargle frequently, keep their mouths clean, and avoid consumption of hot, hard, and spicy food.

\section{Blood study}

After acquiring consents from parents, 4-mL peripheral venous blood samples was collected from each fasting child in the morning of day 2 before the operation and 1 and 3 months after the operation. Aliquots of the blood samples were then transferred to heparin anticoagulation tubes and were used to determine the percentage of various T-cell subpopulations and the levels of Igs. A flow cytometry analyzer (BD FACScan flow cytometry, Becton Dickinson, USA) was used to determine the percentage of T-lymphocyte subsets of $\mathrm{CD}^{+}$, $\mathrm{CD}^{+}$, and $\mathrm{CD}^{+}$, and then, the $\mathrm{CD} 4^{+} / \mathrm{CD}^{+}$ratio was calculated (the reagents used in the process were provided by BD Inc., USA). The levels of IgA, IgG, and IgM were determined with a specific protein detector (Array 360 specific protein analyzer; Beckman Coulter, Inc., USA); the monoclonal antibodies were provided by Beckman Coulter, Inc. During the test, the children did not receive any drugs affecting immune function.

\section{Statistical analysis}

The SPSS 13.0 software was used for statistical analysis, in which the paired $t$ test was used with the parameters reported by means $\pm \mathrm{SD}$, and $\mathrm{P}<0.05$ considered to be statistically significant.

\section{RESULTS}

\section{Humoral immunity}

In the complete resection group, the levels of $\operatorname{IgA}, \operatorname{IgG}$, and $\operatorname{IgM} 1$ month after the operation were significantly decreased from those before the operation; these levels returned to the pre-operative levels 3 months after the operation. These changes were statistically significant $(\mathrm{P}<0.01$; Table 1$)$. In the partial resection group, the level of humoral immunity was slightly decreased, but this difference was not statistically significant $(\mathrm{P}>0.05$; Table 1$)$.

\section{Cellular immunity}

Table 1. Comparison of serum IgA, IgG, and IgM before and after the operation.

\begin{tabular}{|c|c|c|c|c|c|c|}
\hline \multirow[t]{2}{*}{ Groups } & \multicolumn{2}{|c|}{ IgA } & \multicolumn{2}{|c|}{ IgG } & \multicolumn{2}{|c|}{$\operatorname{IgM}$} \\
\hline & $\begin{array}{c}\text { Partial } \\
\text { tonsillectomy }\end{array}$ & $\begin{array}{c}\text { Complete } \\
\text { tonsillectomy }\end{array}$ & $\begin{array}{c}\text { Partial } \\
\text { tonsillectomy }\end{array}$ & $\begin{array}{c}\text { Complete } \\
\text { tonsillectomy }\end{array}$ & $\begin{array}{c}\text { Partial } \\
\text { tonsillectomy }\end{array}$ & $\begin{array}{c}\text { Complete } \\
\text { tonsillectomy }\end{array}$ \\
\hline Pre-operation & $1.64 \pm 0.72$ & $1.69 \pm 0.67$ & $11.62 \pm 1.76$ & $11.54 \pm 2.38$ & $1.40 \pm 0.49$ & $1.42 \pm 0.53$ \\
\hline One month after operation & $1.47 \pm 0.58$ & $1.09 \pm 0.27 *$ & $10.94 \pm 2.14$ & $9.75 \pm 2.39 *$ & $1.36 \pm 0.52$ & $0.96 \pm 0.57 *$ \\
\hline Three months after operation & $1.65 \pm 0.55$ & $1.61 \pm 0.59$ & $11.01 \pm 2.78$ & $11.71 \pm 2.64$ & $1.61 \pm 0.47$ & $1.29 \pm 0.65$ \\
\hline
\end{tabular}

$* \mathrm{P}<0.01$, after 1 month, the levels of $\operatorname{IgA}, \operatorname{IgG}, \operatorname{IgM}$ of the complete resection group prior to the operation were compared with those after the operation and the changes were all statistically significant. 
The $\mathrm{CD}^{+} / \mathrm{CD}^{+}$ratio for both groups showed a slight decrease after the operation and it returned to the baseline level 3 months after the operation; however, these differences were not statistically significant ( $\mathrm{P}>0.05$; Table 2$)$; for both groups, the changes in the levels of $\mathrm{CD}^{+}, \mathrm{CD}^{+}$, and $\mathrm{CD} 8^{+}$after the operation (compared to that before the operation) were not statistically significant $(\mathrm{P}>0.05$; Table 2$)$.

\begin{tabular}{|c|c|c|c|c|}
\hline \multirow[t]{2}{*}{ Groups } & \multicolumn{2}{|c|}{$\mathrm{CD}^{+}$} & \multicolumn{2}{|c|}{$\mathrm{CD}^{+}{ }^{+}$} \\
\hline & Partial tonsillectomy & Complete tonsillectomy & Partial tonsillectomy & Complete tonsillectomy \\
\hline Pre-operation & $69.65 \pm 4.44$ & $68.42 \pm 4.14$ & $32.66 \pm 6.59$ & $33.84 \pm 6.71$ \\
\hline One month after operation & $67.91 \pm 7.04$ & $68.48 \pm 6.44$ & $31.92 \pm 5.94$ & $32.08 \pm 5.46$ \\
\hline \multirow[t]{6}{*}{ Three months after operation } & on $65.24 \pm 6.58$ & $66.38 \pm 5.67$ & $32.36 \pm 5.67$ & $34.76 \pm 5.27$ \\
\hline & \multicolumn{2}{|c|}{$\mathrm{CD}^{+}$} & \multicolumn{2}{|c|}{$\mathrm{CD}^{+} / \mathrm{CD}^{+}$} \\
\hline & Partial tonsillectomy & Complete tonsillectomy & Partial tonsillectomy & Complete tonsillectomy \\
\hline & $24.55 \pm 4.30$ & $25.35 \pm 4.51$ & $1.39 \pm 0.33$ & $1.43 \pm 0.42$ \\
\hline & $24.74 \pm 4.22$ & $26.57 \pm 3.52$ & $1.34 \pm 0.35$ & $1.25 \pm 0.26$ \\
\hline & $23.08 \pm 4.10$ & $24.21 \pm 4.17$ & $1.45 \pm 0.37$ & $1.49 \pm 0.41$ \\
\hline
\end{tabular}

\section{DISCUSSION}

In severe conditions, children with OSAHS may have retarded growth and development, craniofacial abnormalities (Flores-Mir et al., 2013), cognitive impairment, and cardiovascular system diseases (Bhattacharjee et al., 2009) because of nocturnal recurrence of apnea and hypopnea and a more frequent and persistent reduction of $\mathrm{SpO}_{2}$ during the rapid eye movement phase of sleep. The classical risk factor for OSAHS in normal weight children is enlargement of the adenoids and tonsils, which differs from that in adult patients. Arens et al. (2001) showed that in sedated children, a correlation exists between increased tonsil and adenoid volume and AHI. Fregosi et al. (2003) obtained similar results in slightly older, unsedated children, with a correlation between increasing tonsil cross-sectional area (CSA), soft palate CSA, and obstructive AHI. Arens et al. (2005) found that even in asymptomatic children, an increased adenotonsillar size did correlate with increased nasopharyngeal airway narrowing, that is, increased tissue compliance, during inspiration.

Adenotonsillectomy resulted in an increase in the airway CSA of the retropalatal pharynx, leading to approximately 2 -fold reduction in the axial velocities in this region and an increase in static pressure (Vos et al., 2010). Therefore, adenotonsillectomy is the most classic operating approach, and hypertrophic tonsil handling is an unavoidable issue in the surgical treatment of childhood OSAHS.

As an important part of the Waldeyer's ring, the tonsil, located at the common entrance of the gastrointestinal and respiratory tracts, is the most frontline barrier for contact with the inhaled and ingested antigens. In addition to its role as a secondary lymph organ, tonsils also have a special crypt system, which consists of a complex network of pipes and can retain passing antigens for disposal by the immune system, and then transport these antigens to the immunocompetent cells under the tonsil epithelial cells (Ivarsson et al., 1999). Thus, the tonsil is a part of the mucosa-associated lymphoid tissue, with roles in both humoral and cellular immunity. The humoral immunity depends on B cells, plasma cells, and various antibodies; 
when B lymphocytes are stimulated by antigens, they proliferate and evolve into plasma cells, resisting the antigen and producing antibodies. When $\mathrm{T}$ cells in the body of a child are stimulated by antigens, they will differentiate into activated lymphocytes, and among them, some will become T helper cells (CD4) or T cytotoxic cells (CD8). CD4 cells can stimulate the B cells to transform into plasma cells (antibody-producing cells) and CD8 cells can kill the antigen-containing cells by direct contact and cytokine release (Quiding-Järbrink et al., 1995). In normal situations, the ratio of $\mathrm{CD}^{+}$and $\mathrm{CD} 8^{+}$is somewhat stable, and they are affected and antagonized by each other to maintain the balance of the immune system. When the immune function is decreased, it is usually manifested by the shortage of CD4 subsets and/ or the increase of CD8 subsets, or the decrease of CD4/CD8 ratio.

Because of the incomplete development of the systemic immune organs in childhood, the tonsil immune activity is more important in children than in adults. The tonsils and adenoids in children have the function of producing lymphocytes and non-lymphocytes such as $\mathrm{T}$ lymphocytes, B lymphocytes, dendritic cells, and $\mathrm{M}$ cells, and the number of lymphocytes is significantly higher than that in adults, and these cells work synergistically to complete the immunization process together. Tonsils and adenoids can also secrete cytokines and IgA, IgG, and IgM. In the submucosa, the number of B cells is higher than the number of T cells in the lymphoid tissue, and most of them can produce secretory immunoglobulin $\mathrm{A}$ (SIgA), which is an important factor for the local mucosal immunity against infection.

Tonsil adenoidectomy is a common method for the treatment of children with OSAHS, but scholars have different opinions on its effects on immune functions. Zielnik-Jurkiewicz and Jurkiewicz (2002) found that the children with tonsil adenoidal hypertrophy have significantly higher levels of $\operatorname{IgA}$, IgM, and IgG before operation than the normal control group. One month after operation, the level of immunoglobulin was significantly decreased, suggesting humoral immunodeficiency. The levels of $\operatorname{IgA}, \operatorname{IgM}$, and $\operatorname{IgG}$ returned to the normal levels 6 months after operation. For the cellular immune function, the pre-operative number of $\mathrm{CD} 8^{+}$ cells was significantly higher and it constantly decreased after the operation and returned to the same level as the control group. Kaygusuz et al. (2009) found that a month after tonsillectomy, the levels of $\operatorname{IgG}$, IgA, and IgM were significantly decreased compared to that in the age-matched healthy control group, among which, the levels of IgA and IgM was decreased 54 months after the operation. In case of cellular immunity, the numbers of $\mathrm{CD} 4^{+}$and $\mathrm{CD} 19^{+}$ cells in the children were significantly decreased and the numbers of $\mathrm{CD}^{+}, \mathrm{CD} 16^{+}, \mathrm{CD} 65^{+}$, and $\mathrm{CD} 25^{+}$were significantly increased 1 month after the operation and they returned to the same level as the control group 54 months after the operation. Böck et al. (1994) reported that the immune functions were not affected in patients whose tonsils were removed in childhood.

We found that the post-operative levels of $\operatorname{IgA}$, IgM, and IgG in the tonsillectomy groups were significantly decreased from that before the operation, which is consistent with the results of the studies conducted by Zielnik-Jurkiewicz and Jurkiewicz (2002) and Kaygusuz et al. (2009). However, we observed that the levels of $\operatorname{IgA}$, IgM, and IgG were returned to the 3-month levels before the operation, which was earlier than the 6 months required in the study conducted by Zielnik-Jurkiewicz. For the group of partial resection plus ablation, the levels of $\operatorname{IgA}$, IgM, and IgG 1 month after the operation were not significantly changed from that before the operation, suggesting that the remnant tonsils possessed some immune functions and could avoid immune deficiency after the operation. The post-operative changes in the cellular immunity of both groups had no statistical significance either, which was con- 
sistent with the conclusion obtained by Böck et al. (1994). Because we did not set the control group in the study, it was impossible to compare the immune function in children before and after the operation and to validate the conclusion obtained by Zielnik-Jurkiewicz and Jurkiewicz (2002) and Kaygusuz et al. (2009).

Low-temperature plasma radiofrequency ablation has merits such as less hemorrhage, less severe post-operative pain, and less damage to the surrounding tissues. Shah and Dunham (2007) observed the tonsillar wound surface after low-temperature plasma radiofrequency ablation and did not find basophilic substance invasion at the verge of the tonsil, tissue carbonization, or damage at the depth of the tonsil crypt. In this study, the results showed that tonsil partial resection plus ablation by using the low-temperature plasma technique did not cause marked changes in the immune function of the tonsils within a short period after the surgery. This finding indicates that low-temperature plasma causes minor tissue damage. After operation, the epithelial basic structure, cellular levels, and immune function of the tonsillar crypts are only slightly damaged and the immune function of the tonsils does not noticeably decrease. Therefore, it is predictable that the residual tonsils after operation still secrete cytokines and immunoglobulins, possibly sIgA, which possess local mucosal immunity against infection and can therefore serve as the first line of defense of the respiratory system. This is of particular significance for children with incomplete immune function accompanied by adenoid deletion. Furthermore, the residual tonsils may effectively avoid post-operative complications such as pharyngeal foreign body sensation and pharyngoxerosis.

Low-temperature radiofrequency plasma ablation is becoming increasingly prevalent in recent years for ENT applications. Technically, low-temperature radiofrequency plasma ablation can be used for partial resection of tonsils with hypertrophy of grade III; however, there is a possibility that the immune function of the tonsils will be suppressed because of the destruction of the anatomic structure. Our study confirmed that partial resection can preserve partial tonsil tissue, and especially, a part of the immune function of the tonsils while relieving the airway obstruction, thus avoiding the destruction of the protective barrier or the impairment of respiratory defense mechanisms, and avoiding the early humoral immune deficiency. However, this study has some limitations. The number of cases included should be increased, the immune functions of the patients as well as the local immune function of the tonsils should be monitored before operation, and the long-term pharyngeal foreign body sensation and pharyngoxerosis should be observed after operation.

\section{REFERENCES}

Abbey K and Kawabata I (1988). Computerized three-dimensional reconstruction of the crypt system of the palatine tonsil. Acta Otolaryngol. Suppl. 454: 39-42.

Arens R, McDonough JM, Costarino AT, Mahboubi S, et al. (2001). Magnetic resonance imaging of the upper airway structure of children with obstructive sleep apnea syndrome. Am. J. Respir. Crit. Care Med. 164: 698-703.

Arens R, Sin S, McDonough JM, Palmer JM, et al. (2005). Changes in upper airway size during tidal breathing in children with obstructive sleep apnea syndrome. Am. J. Respir. Crit. Care Med. 171: 1298-1304.

Bhattacharjee R, Kheirandish-Gozal L, Pillar G and Gozal D (2009). Cardiovascular complications of obstructive sleep apnea syndrome: evidence from children. Prog. Cardiovasc. Dis. 51: 416-433.

Böck A, Popp W and Herkner KR (1994). Tonsillectomy and the immune system: a long-term follow up comparison between tonsillectomized and non-tonsillectomized children. Eur. Arch. Otorhinolaryngol. 251: 423-427.

Brietzke SE and Gallagher D (2006). The effectiveness of tonsillectomy and adenoidectomy in the treatment of pediatric obstructive sleep apnea/hypopnea syndrome: a meta-analysis. Otolaryngol. Head Neck Surg. 134: 979-984.

Flores-Mir C, Korayem M, Heo G, Witmans M, et al. (2013). Craniofacial morphological characteristics in children with 
obstructive sleep apnea syndrome: a systematic review and meta-analysis. J. Am. Dent. Assoc. 144: 269-277.

Fregosi RF, Quan SF, Kaemingk KL, Morgan WJ, et al. (2003). Sleep-disordered breathing, pharyngeal size and soft tissue anatomy in children. J. Appl. Physiol. 95: 2030-2038.

Fujioka M, Young LW and Girdany BR (1979). Radiographic evaluation of adenoidal size in children: adenoidalnasopharyngeal ratio. AJR Am. J. Roentgenol. 133: 401-404.

Goldbart AD, Krishna J, Li RC, Serpero LD, et al. (2006). Inflammatory mediators in exhaled breath condensate of children with obstructive sleep apnea syndrome. Chest 130: 143-148.

Ivarsson M, Quiding-Järbrink M and Lundberg C (1999). Immunoglobulin-secreting cells in the surface secretion on the pharyngeal tonsils. Acta Otolaryngol. 119: 939-943.

Kaygusuz I, Alpay HC, Godekmerdan A, Karlidag T, et al. (2009). Evaluation of long-term impacts of tonsillectomy on immune functions of children: a follow-up study. Int. J. Pediatr. Otorhinolaryngol. 73: 445-449.

Lal C, Strange C and Bachman D (2012). Neurocognitive impairment in obstructive sleep apnea. Chest 141: 1601-1610.

Landau YE, Bar-Yishay O, Greenberg-Dotan S, Goldbart AD, et al. (2012). Impaired behavioral and neurocognitive function in preschool children with obstructive sleep apnea. Pediatr. Pulmonol. 47: 180-188.

Lim J and McKean MC (2009). Adenotonsillectomy for obstructive sleep apnoea in children. Cochrane Database Syst. Rev. CD003136.

Marcus CL, Brooks LJ, Draper KA, Gozal D, et al. (2012). Diagnosis and management of childhood obstructive sleep apnea syndrome. Pediatrics 130: 576-584.

Quiding-Järbrink M, Granström G, Nordström I, Holmgren J, et al. (1995). Induction of compartmentalized B-cell responses in human tonsils. Infect. Immun. 63: 853-857.

Shah UK and Dunham B (2007). Coblation for tonsillectomy: an evidence-based review. ORL J. Otorhinolaryngol. Relat. Spec. 69: 349-357.

The AASM Manual for the Scoring of Sleep and Associated Events (2007). Rules, Terminology and Technical Specifications. AASM Manual for Scoring Sleep, Westchester.

Vos WG, De Backer WA and Verhulst SL (2010). Correlation between the severity of sleep apnea and upper airway morphology in pediatric and adult patients. Curr. Opin. Allergy Clin. Immunol. 10: 26-33.

Zielnik-Jurkiewicz B and Jurkiewicz D (2002). Implication of immunological abnormalities after adenotonsillotomy. Int. J. Pediatr. Otorhinolaryngol. 64: 127-132. 